\title{
Retroperitoneal Bronchogenic Cyst Treated by Open Surgery: A Rare Case Report
}

\author{
Ramzi Mejri ${ }^{1 *}$, Khaireddine Mrad Dali², Kays Chaker ${ }^{2}$,Bibi Mokhtar ${ }^{2}$, Sami Ben Rhouma ${ }^{2}$ \\ and Yassine Nouira ${ }^{2}$
}

${ }^{1}$ Department of Urology, Hospital Mongi Slim La Marsa, Tunisia

${ }^{2}$ Department of Urology, La Rabta Hospital, Tunisia

\begin{abstract}
Bronchogenic cyst is considered to be a benign morphological abnormality in the development of the primitive foregut, which usually occurs in the thorax, particularly in the mediastinum. Subdiaphragmatic and retroperitoneal locations in particular are unusual. It is usually asymptomatic. Clinical presentations are related to complicated forms (infection, hemorrhage and extrinsic compression). Imaging rarely contributes to the diagnosis. Only histopathological examination allows a definitive diagnosis of retroperitoneal bronchogenic cyst. Surgical resection is the therapeutic strategy of choice. We report a peculiar presentation of a symptomatic retroperitoneal bronchogenic cyst that can be confused with adrenal masses.
\end{abstract}

\section{Keywords}

Bronchogenic cyst, Retroperitoneum, Adrenal gland, Surgical resection

\section{Introduction}

Bronchogenic cysts are rare benign congenital anomalies that result from aberrant budding of the tracheobronchial tree. Usually, these bronchogenic cysts remain attached to the primitive foregut and thus attached to the tracheobronchial tree. However, in rare cases, they may migrate and separate from the respiratory tract to various atypical locations, including the neck, spinal, subphrenic and even retroperitoneal regions [1]. The majority of cases reported in the literature have been diagnosed incidentally. In case of infection, perforation or extrinsic compression of bronchogenic cysts, telling clinical manifestations have occurred. The uncertainty of the diagnosis after radiological explorations supports the surgical indication in view of a conclusive histological assessment. The present study presents the original case of a retroperitoneal bronchogenic cyst in intimate contact with the left subdiaphragmatic region that was successfully treated by open retroperitoneal surgery. The clinical, radiological, anatomopathological data and therapeutic conduct are reported and analyzed in this case.

\section{Case Report}

A 51-year-old man presented to our urological surgery unit complaining of pain in the left flank and epigastric region associated with vomiting and febrile episodes. The patient also reports anorexia and asthenia without any notion of weight loss. His medical history included well-balanced diabetes mellitus, arterial hypertension and chronic spinal arthralgia. This symptomatology has been evolving intermittently for a month with exacerbation in the postprandial period or after intense physical activity. The clinical examination was unremarkable. Laboratory tests showed a high level of inflammatory markers (CRP $85 \mathrm{mg} / \mathrm{l}$ and leukocytes 11200/ $\mathrm{mm}^{3}$ ), a correct pancreatic balance (normal lip asemia and amyl asemia) and normal renal function. The hormonal balance of the adrenal gland and the hydatid serology are also normal. Abdominal ultrasound revealed a hypoechoic cystic formation 5 centimeters in diameter extending to the left adrenal region. Ultrasound exploration was hampered by the interposition of digestive gases and the depth of the lesion. In order to support the diagnosis, a thoracoabdomino pelvic computed tomography scan was performed showing a well-limited oval mass of $5.3 \mathrm{~cm}$ long axis, in contact with the left diaphragm pillar (Figure 1a). This formation presents a para-liquid density, not enhanced after injection of the

*Corresponding author: Ramzi Mejri, Department of Urology, Hospital Mongi Slim La Marsa, Tunisia, Tel: 21698641685

Accepted: October 29, 2021

Published online: October 31, 2021

Citation: Mejri R, Dali KM, Chaker K, et al. (2021) Retroperitoneal Bronchogenic Cyst Treated by Open Surgery: A Rare Case Report. Ann Endocrinol Metab 4(1):73-76 


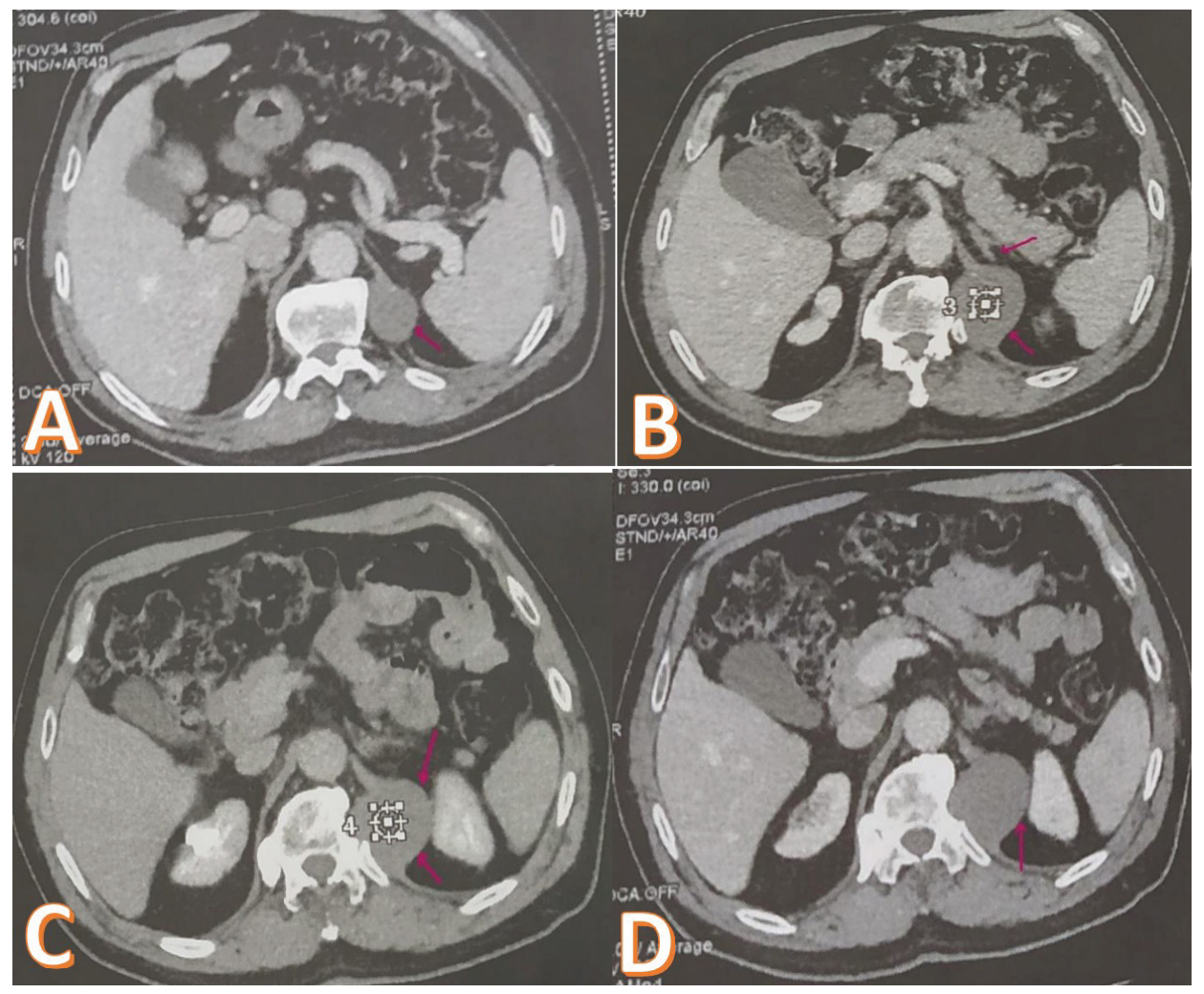

Figure 1: Thoracoabdominopelvic Computed tomography; A) Well-limited oval mass of $5.3 \mathrm{~cm}$ long axis in contact with the pillar of the left diaphragm; B) Parafluidic density mass not taking up contrast in contact with the adrenal gland; C,D) Cystic formation in contact with the left kidney.

contrast medium. It is in contact with the $12^{\text {th }}$ dorsal vertebra and the homolateral costo-vertebral joint, without any associated bone lesion (Figure 2). However, this mass bulges into the retroperitoneum and appears to remain distant from the kidney and the left adrenal gland (Figure 1b, Figure $1 \mathrm{c}$ and Figure 1d). Given the diagnostic uncertainty and the suspicion of a cystic tumor of the adrenal gland, we opted for an elective posterior and retroperitoneal surgical approach due to the unavailability of laparoscopy. Intraoperative exploration showed an encapsulated cystic lesion with smooth, clean and easily dissected borders in contact with the left diaphragm pillar and the posterior part of the left adrenal gland. The entire mass was resected with the adherent part of the left adrenal gland. The patient was discharged on the fifth day after a favorable clinical course. Histopathological study showed ciliated respiratory columnar epithelium associated with seromucosal acini and cartilage islands (Figure 3). The adrenal gland was histologically healthy. These histological findings were in favor of a bronchogenic cyst. The patient is regularly followed up as an outpatient, he was in good physical and mental health, his renal function was normal but he kept spinal pain during the first months in connection with the surgical approach. After a two-year follow-up, no cystic recurrence was reported.

\section{Discussion}

Bronchogenic cysts are alterations in the development of the foregut early in gestational life. The majority of bronchogenic cysts are located in the mediastinum and only $15 \%$ originate in the lung parenchyma [2]. Occasionally, they can occur in many atypical areas, ranging from the neck to the spinal dura and even below the diaphragm. Its preferred location when it is retroperitoneal is the midline near the spleen, the pancreas and the left adrenal gland as in our present case. The pathogenesis is not yet elucidated, but it has been reported that a defect in the closure of the pleuroperitoneal duct in association with abnormal budding of the primitive foregut may induce migration of the tracheobronchial tree bud into the retroperitoneal cavity. Retroperitoneal bronchogenic cysts are usually diagnosed incidentally and are mostly asymptomatic. Symptomatic forms are usually related to secondary complications such as infections, perforation, hemorrhage, and extrinsic compressions of adjacent structures.

Thus, they may be confused with a pheochromocytoma because of the pressure exerted near the adrenal gland. Patients have reported more subtle symptoms, such as vague complaints of abdominal discomfort and early satiety [3]. Due to an atypical clinical manifestation, retroperitoneal bronchogenic cysts are often detected incidentally and diagnosed by radiological exploration procedures such as computed tomography or magnetic resonance imaging. On computed tomography, bronchogenic cysts are manifested as round, well-limited hypodense formations without enhancement arising in the subdiaphragmatic space [1]. Early surgical resection of cystic and solid retroperitoneal 


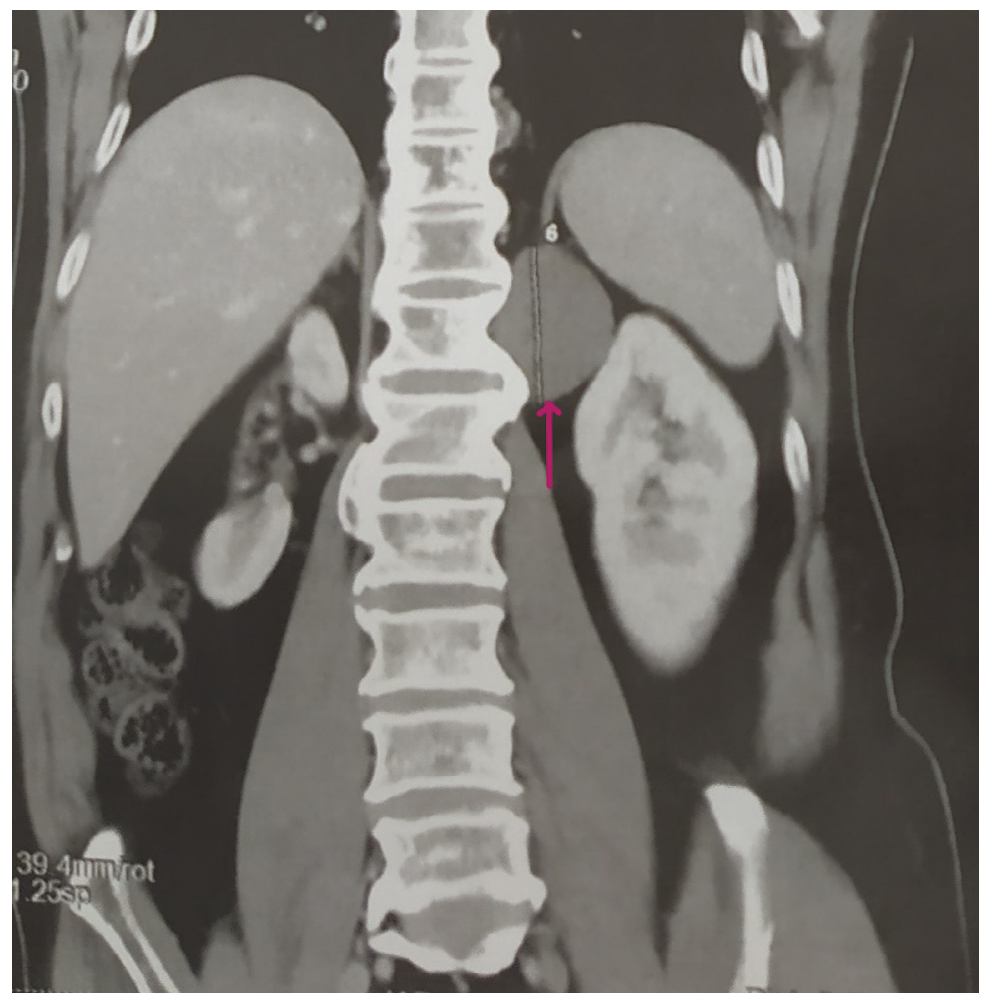

Figure 2: Thoracoabdominopelvic Computed tomography: Reconstruction image showing a cystic mass embedded between the spleen, the adrenal gland and the left kidney and located at the level of the $12^{\text {th }}$ rib.

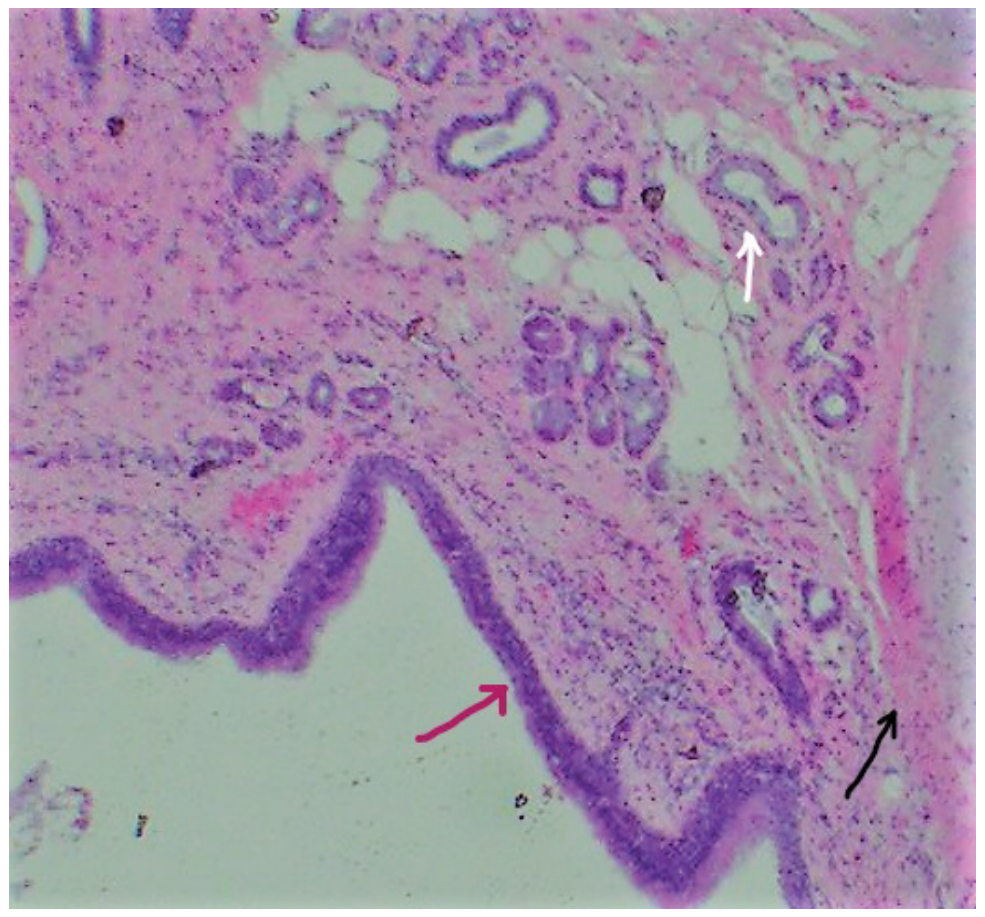

Figure 3: Histological examination of Bronchogenic cyst (Hematoxylin and eosin staining; 20x magnification). The cyst wall lined with ciliated columnar epithelium (red arrow), bundles of smooth muscles (black arrow) and seromucous glands (white arrow).

masses especially at the asymptomatic stage is strongly recommended. This therapeutic approach is based on the one hand on the infectious risk of cysts complicating their removal and on the other hand on the uncertainty of a preoperative diagnosis. The surgical approach will be determined by the characteristics and location of the lesion. Attachment to neighboring organs may require total resection of these lesions as was the case in our patient. According to recent data in the 
literature, retroperitoneoscopy is an efficient, less invasive and valid surgical technique [4]. If a retroperitoneoscopic approach is not available, a posterior open elective approach can be used. If the cyst has adhered to neighboring organs, its residual wall must be cauterized by electrocoagulation to avoid any risk of recurrence.

\section{Conclusion}

Bronchogenic cysts are benign congenital malformations of the foregut caused by ectopic migration of bronchial buds. Its presence in more distant locations, such as the retroperitoneum, is extremely rare. Only about 60 cases have been reported in the literature. They pose the problem of differential diagnosis of cystic and solid retroperitoneal masses, with a predilection for location in the left upper quadrant. Surgical resection of these lesions is the rule in order to alleviate symptoms, prevent complications, and establish a definitive positive diagnosis based on histopathologic data.

\section{Competing Interests}

The authors declare that there are no conflicts of interest regarding the publication of this article.

\section{Authors Contributions}

$\mathrm{R}$ Mejri: Participated in the writing of the manuscript; $\mathrm{K}$ Mrad Dali: Participated in the writing of the manuscript; $K$ Chaker: Participated in the writing of the manuscript; $M$ Bibi: Participated in the writing of the manuscript; $S$ Ben Rhouma: Participated in the writing of the manuscript and its correction; $Y$ Nouira: Participated in the writing of the manuscript and its correction.

\section{References}

1. Buckley JA, Siegelman ES, Birnbaum BA, et al. (1998) Bronchogenic cyst appearing as a retroperitoneal mass. AJR Am J Roentgenol 171: 527-528.

2. Rapado F, Bennett JD, Stringfellow JM (1998) Bronchogenic cyst: An unusual cause of lump in the neck. J Laryngol Otol 112: 893894.

3. Wang SE, Tsai YF, Su CH, et al. (2006) Retroperitoneal bronchogenic cyst mimicking pancreatic cystic lesion. J Chin Med Assoc 69: 538-542.

4. Ishikawa T, Kawabata G, Okada H, et al. (2003) Retroperitoneal bronchogenic cyst managed with retroperitoneoscopic surgery. J Urol 169: 1078-1079.

DOI: $10.36959 / 433 / 570$ 